Article

\title{
External Field-Controlled Ablation: Magnetic Fields
}

\author{
Jovan Maksimovic ${ }^{1,2 *}$, Soon Hock $\mathrm{Ng}^{1,2}$, Tomas Katkus ${ }^{1}$, Bruce C. C. Cowie ${ }^{2}$ and Saulius \\ Juodkazis ${ }^{1,3,4 *}$ \\ Swinburne University of Technology, John st., Hawthorn, Vic 3122, Australia \\ Australian Synchrotron, 800 Blackburn Road, Clayton, Vic 3168, Australia \\ Melbourne Center for Nanofabrication, Australian National Fabrication Facility, Clayton, Vic 3168, Australia \\ 4 Tokyo Tech World Research Hub Initiative (WRHI), School of Materials and Chemical Technology, Tokyo \\ Institute of Technology, 2-12-1, Ookayama, Meguro-ku, Tokyo 152-8550, Japan \\ *Corresponding: jmaksimovic@swin.edu.au; saulius.juodkazis@gmail.com
}

\begin{abstract}
Laser ablation of silicon under an external applied magnetic field with different orientations was investigated in respect to the scanning direction and polarisation of the laser beam, by observation of the ablation patterns and debris deposition. Ultra-short $\sim 230$ fs laser pulses of $1030 \mathrm{~nm}$ wavelengths were used in the single and multi-pulse irradiation modes. Ablation with an externally applied magnetic B-field $B_{\text {ext }} \approx 0.1 \mathrm{~T}$ is shown to strongly affect debris formation. The mechanism of surface plasmon polariton (SPP) wave can explain the ablated periodic patterns observed with alignment along the magnetic field lines. Application potential of an external field controlled ablation is discussed.
\end{abstract}

Keywords: ablation; magnetic field; femtosecond laser fabrication

\section{Introduction}

Ultra-short laser pulses provide nanoscale resolution in 3D polymerisation, optical waveguide inscription in glasses and crystals [1], non-erasable optical memory and photonic crystals [2-4], nano-structuring of surfaces and in the bulk [5-8], creation of new materials and their high pressure/temperature phases by 3D confined micro-explosions [9-11], thermal morphing of laser fabricated 3D structures [12], and laser assisted etching [13-15]. Applications of coloidal nanoparticle synthesis by ablation in liquids [16] and laser machining have become industrial applications with a high throughput [17-20].

Here, we introduce a new control method over the ablation process using an externally applied magnetic B-field. With a Si sample placed in a millimeter-scale gap between Nd-magnets of $\sim 0.1 \mathrm{~T}$, the ablation plume, as well as the ablated surface of crystalline $\mathrm{Si}$, on which a surface plasmon polariton (SPP) wave has been induced, were strongly affected. The orientation of magnetic B-field rather than the polarisation of the laser light's E-field strongly governs the formation of the imprinted patterns of aligned micro-ablation pits. The laser ablated patterns are determined by the instant electron densities in the surface layer, their velocity and magnetic field strength.

\section{Results}

\subsection{Peculiarities of ablation patterns in the external B-field}

Figure 1 shows the observed morphology change of single-pulse ablation sites on $\mathrm{Si}$ in the presence of an externally applied magnetic field $B_{\text {ext }}=0.1 \mathrm{~T}$. Ablation was carried out at a high intensity, typical for laser cutting applications, exceeding the ablation threshold more than ten times. The direction of ablation plume and polarisation of light E-field were both perpendicular to the magnetic field to 


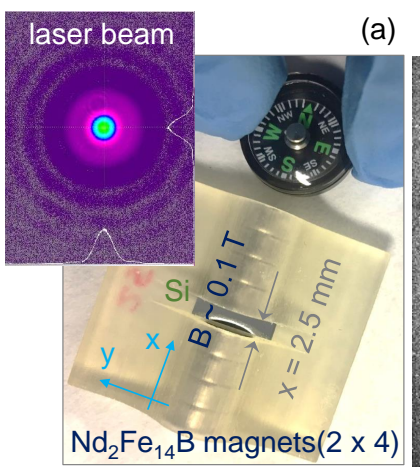

a)

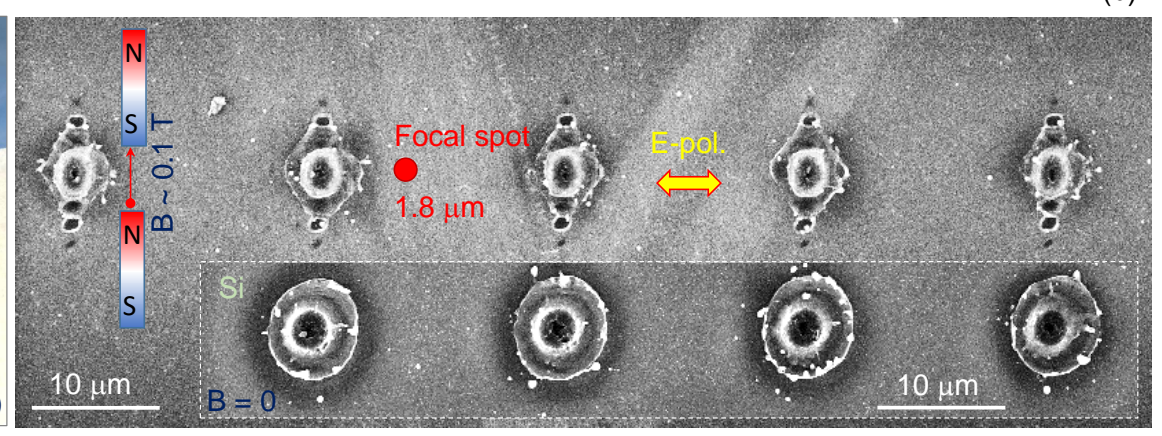

Figure 1. (a) 3D printed jig with a sample compartment gap of $x=2.5 \mathrm{~mm}$ surrounded by Nd-magnets (four on each side). A (100) orientation Silicon wafer was diced along the (110) plane to the required width; magnetic field was oriented along $\langle\overline{1} 10\rangle$ direction (perpendicular to the wafer base cut $\langle 110\rangle$ ). The inset shows Gaussian beam profile of femtosecond laser used. (b) Scanning electron microscopy (SEM) images of single pulse ablated regions with and without magnetic fields present. Laser pulses of $\lambda=1030 \mathrm{~nm}$ wavelength and $t_{p}=230 \mathrm{fs}$ pulse duration were focused with an objective lens of numerical aperture $N A=0.7$ to a focal spot of $2 r=1.22 \lambda / N A=1.8 \mu \mathrm{m}$ diameter; pulse energy was $E_{p}=1.13 \mu \mathrm{J}$ at the entrance of the objective lens (transmission of the lens was $T=0.22$ ), fluence $F_{p} \equiv E_{p} / A_{p}=9.8 \mathrm{~J} / \mathrm{cm}^{2}$, irradiance $I_{p} \equiv F_{p} / t_{p}=42.7 \mathrm{TW} / \mathrm{cm}^{2}$ with area $A_{p}=\pi r^{2}$. For the tilted-SEM view see Supplement Figure A1.

maximise the Lorenz force $\mathbf{F}=q \mathbf{E}+q[\mathbf{v} \times \mathbf{B}]$ for charges $q$ in the ablation plume. Patterns of periodic small ablation pits were formed along the applied B-field (on $\mathrm{Si}\langle\overline{1} 10\rangle$ ) extending well beyond the focal spot diameter. Separation between the adjacent pits were close to the wavelength of laser pulse and the surface plasmon polariton (SPP) at the Si-air interface. Without a magnetic field present $B=0$, a circularly symmetric ablation pit was formed characteristic of the measured Gaussian laser beam profile shown in Figure 1. Distinction between a single pulse ablation sites for the $B=0$ and $B \neq 0$ cases are well expressed for every pulse irradiated between the magnets or even in a close proximity of magnets.

A rhomboidal structure around the central elliptically elongated ablation pit has an approximate side orientation perpendicular to the Si directions $\langle 010\rangle$ and $\langle 100\rangle$. The Lorenz force acting on electrons is directed in (out) to the samples surface when the linearly polarised light field is along the $y$-axis $\left(\mathbf{v}_{y} \propto \mathbf{E}_{y}\right)$ while the B-field is perpendicular along the $\mathbf{x}$-axis ( $\mathbf{B}_{x} ;$ Figure 1$)$. In this geometry, free and bound electrons follow an oscillation normal to the Si surface following $\mathbf{F}_{z}$. It was demonstrated that surface ablation in the direction of E-field polarisation when it is perpendicular to the surface is the most efficient in material removal [21]. Ablation pits extending beyond the focal spot of $2 r=1.8 \mu \mathrm{m}$ can be explained by the SPP mechanism as discussed below (Sec. 3).

Figure 2 presents a summary of the observed dependencies of the ablation pattern vs. the gap size $x$, light polarisation (E-field), magnetic B-field orientation and its strength (the gap size $x$ ). The most pronounced orientation dependence is a linearly aligned pattern of ablated circular pits along the B-field orientation. The period of the pattern was changing almost linearly from $\Lambda=1.22 \mu \mathrm{m}$ for the gap $x=5 \mathrm{~mm}$ to $2.3 \mu \mathrm{m}$ for $x=2 \mathrm{~mm}$ (Figure 2). When the gap between magnets was larger than $5 \mathrm{~mm}$, the pattern of linearly aligned micro-pits disappeared and became similar to pulses without applied magnetic fields (magnets removed from the holder). Figure 3 shows a detailed morphology evolution of the ablated sites for different orientations of the linearly polarised fs-laser pulses. The period $\Lambda_{s}$ of the linear pattern of micro-pits aligned to the magnetic B-field is not affected by polarisation of E-field of laser pulse. The effects of a circularly polarised E-field were explored to further detail the alignment of produced patterns, however, the period and alignment were not changed (not shown here for brevity). Differences between the very central region of pulses are due to sensitive alignment between objective and sample surface. 


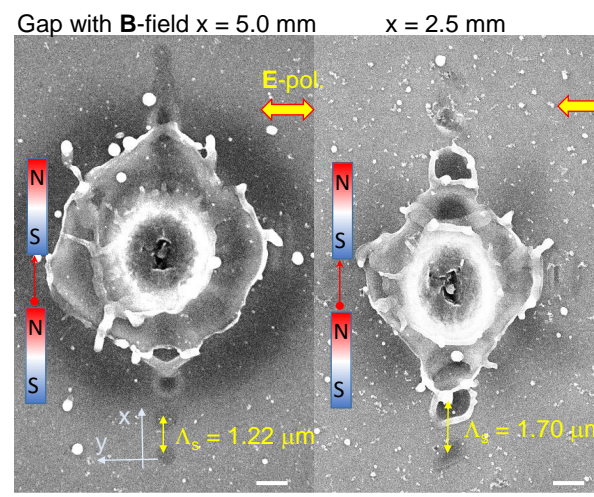

(a)

(b) $\mathrm{x}=2.5 \mathrm{~mm}$ $\mathrm{x}=2.5 \mathrm{~mm}$ $\mathrm{x}=2.0 \mathrm{~mm}$

Figure 2. Summary of separate parameter studies: 1) when gap between magnets $x>5 \mathrm{~mm}$ ablation pattern is barely observed similar to no magnetic field $B=0,2)$ polarisation rotation changes pattern only slightly (b vs c), 3) rotation of magnetic field has strong effect of orientation of SPP pits (c vs d), 4) period $\Lambda_{S}$ of SPP pattern depends on the gap $x$. Pulse energy and focusing conditions like that seen in Fig. 1.
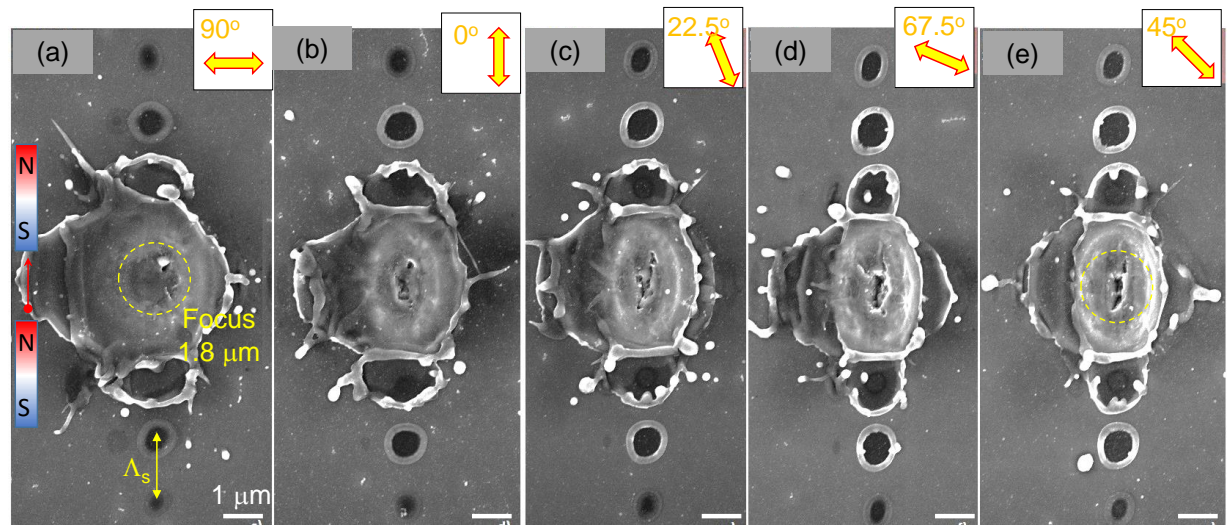

Figure 3. SEM images of the ablation sites under one pulse exposure on Si: the angular polarisation (E-field) dependence (shown in insets). The gap between magnets $x=2.5 \mathrm{~mm}$ and magnetic field $B=0.164 \mathrm{~T}$. The period $\Lambda_{S}$ of SPP pattern was constant. Pulse energy and focusing conditions like that seen in Fig. 1. For the tilted-SEM view see Supplement Figure A1.

Narrow Si samples were tilted inside the magnet gap to check the effect of $B$-field orientation relative to change of the $E$-field orientation of incident light. The pattern of linearly aligned ablation pits was always aligned along the B-field direction while the orientation of the E-field had no effect of the orientation of micro-pits and no coupling was observed.

Patterns of the aligned ablated micro-pits with periodic separation of $1-2 \mu \mathrm{m}$ which depend on the spacing between magnets $x$, resembles a pattern of the SPP wave. SPP has wavelength close to the free space wavelength $\lambda^{s p p} \approx \lambda \sim 1 \mu \mathrm{m}$. The period of ablated pits observed depend on the gap $x$, i.e., on the B-field strength. The linear pattern of ablation pits were also observed at lower pulse energies $E_{p} \approx 10-20 \mathrm{~nJ}$ (on the sample), however, the most distinct features were observed at the highest pulse energy used and presented in Figures 1, 2, 3, A1. Figure 4(a) shows the scaling of the single-pulse ablation site when pulse energy is changed by a factor of $10^{2}$. There was an air breakdown above the Si surface at the utilised pulse energies which were very high and one could expect a change of ablation pattern on the Si surface. However, SEM images in Figure 4(a) shows a continuous change of diameter of the ablation site. The molecular number density of air is $\sim 2.5 \times 10^{19} \mathrm{~cm}^{-3}$ which causes intensity clamping during filamentation in air breakdown by ultra-short laser pulses, however, even full ionisation does not reach the critical plasma density at $1030 \mathrm{~nm}$ wavelength which 
(a)

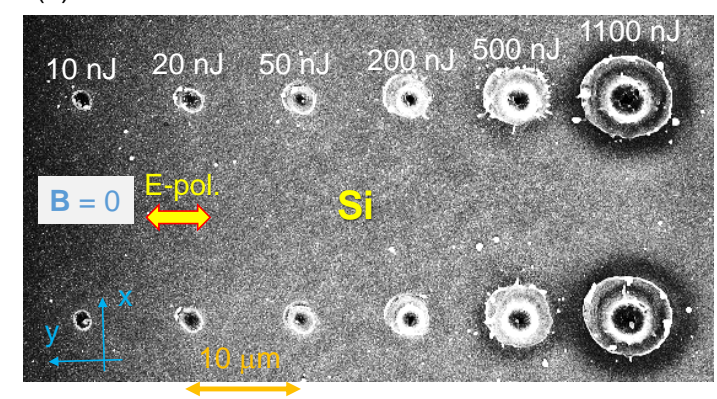

(b)

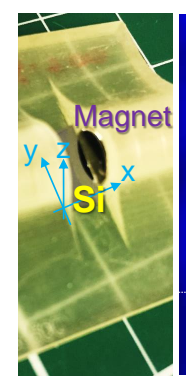

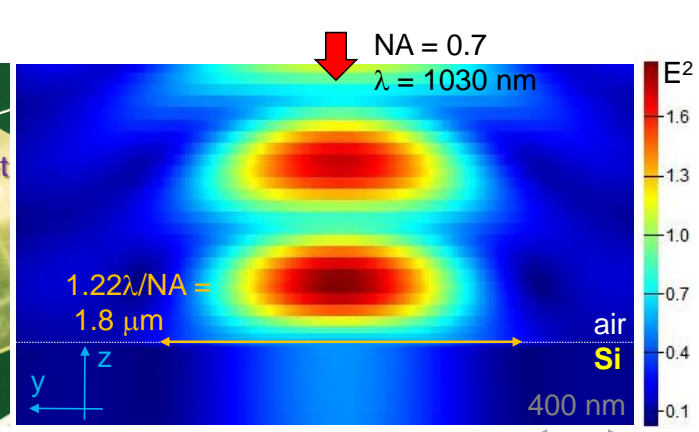

Figure 4. (a) Ablation of $\mathrm{Si}$ at different pulse energies where no magnetic field is applied, $B=0$ (at the entrance of the objective lens of numerical aperture $N A=0.7$; transmission of the objective lens was $T=0.22$ ). For the tilted-SEM view see Supplement Figure A1 and Figure A2. (b) Optical image showing the placement of $\mathrm{Si}$ in the central part of magnetic B-field. Finite difference time domain (FDTD) simulation (Lumerical) of light intensity distribution at the plane of incidence at the conditions of experiments. Modeling presents a linear light intensity distribution. The amplitude of the light source $E_{y}=1$.

is $1.05 \times 10^{21} \mathrm{~cm}^{-3}$. Intensity distribution at the employed $N A=0.7$ focusing is shown in Figure $4(\mathrm{~b})$ for low intensity case.

Figure 5 shows dependence of pulse energy on the linear pattern period $\Lambda_{s}$. Even at low pulse energy (in the tens-of-nJ), the linear pattern aligned to the B-field begins to manifest physically after ablation and as seen in SEM images and evolves into ablation micro-pits at higher energies. The $\Lambda_{s}$ was almost independent of pulse energy $E_{p}$ but follows a close-to-linear dependence on the B-field strength ( $x$ gap width) as shown in Figure 5(b). This is further illustrated by 3-dimensional images in the Appendix. Extrapolation of this dependence shows that the threshold field to observe the linear pattern forming after ablation was $B \approx 0.11 \mathrm{~T}$.

\subsection{Debris dispersion}

When Si samples were placed on a magnetic disk or washer (magnet with a center hole) for ablation, extensive debris formation was observed (Figure 6). When the velocity of the ablation plume $v$ is parallel to the magnetic field $B$, there is no charge separation since the Lorentz force $[\mathbf{v} \times \mathbf{B}]=0$. However, whenever charges start to move at an angle to the magnetic field lines, separation of electrons and ions existing in the laser ablated plume occurs. The charges $\pm|q|$ experience a cyclotron spinning around the B-field lines with a frequency defined by their mass $\omega_{c}=q B / m$. The radius of spinning trajectory is $r_{c}=m v /(q B)$. Apparently, such charge separation in plasma and acquisition of an angular momentum favors longer debris travel times and distances. This favors a longer oxidation time and $\mathrm{SiO}_{2}$ formation which comprises some of the debris on the surface of $\mathrm{Si}$ sample in SEM images (Figure 6).

The near edge X-ray absorption fine structure (NEXAFS) technique was used to identify chemical modification of laser ablated regions. NEXAFS is a useful technique, e.g., to detect phase modifications of olivine [22] at laser irradiation conditions similar to that used in this study. NEXAFS has confirmed an oxide on the surface of laser ablated patterns. Measurements were carried out at the O1s electron binding energy in $\mathrm{SiO}_{2}$ window around $532.9 \mathrm{eV}$ (Soft X-ray beamline of the Australian synchrotron). $\mathrm{Si}$ was ablated with sample placed on the surface of a Nd-magnet the same way as in the experiment on debris generation (Figure 6).

The origin of the newly observed periodic SPP-like pattern of ablated micro-pits aligned to B-field lines is discussed next. 

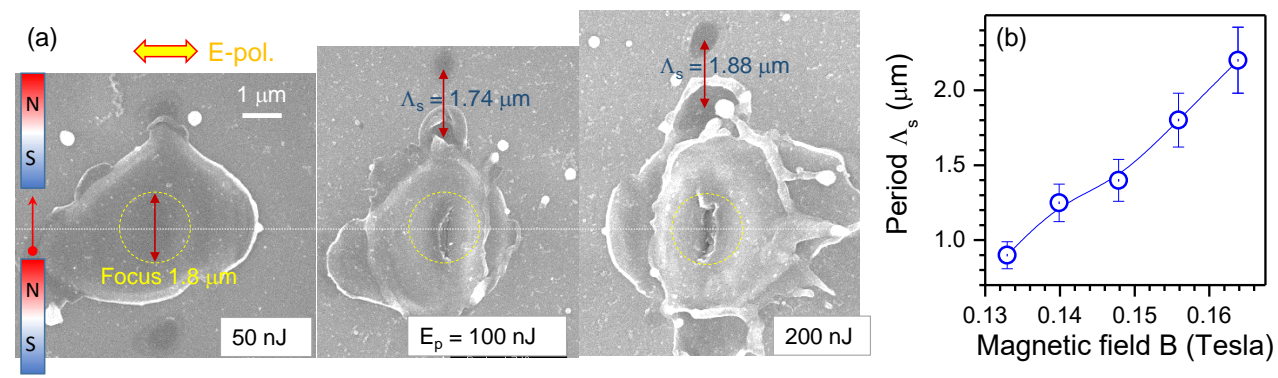

Figure 5. (a) SEM images of single pulse ablation sites at different pulse energies $E_{p}$ (at the entrance of the objective lens of numerical aperture $N A=0.7$; transmission of the objective lens was $T=0.22$ ). The gap $x=2.5 \mathrm{~mm}, B=0.164 \mathrm{~T}$. The arrow markers are the same length of $1.74 \mu \mathrm{m}$ for a better comparison. (b) Period of periodic pattern $\Lambda_{s}$ vs. magnetic field strength (or the gap width $x$ ) at high pulse energy $E_{p}=1.13 \mu \mathrm{J}$. Line is an eye-guide; error bars are $10 \%$.

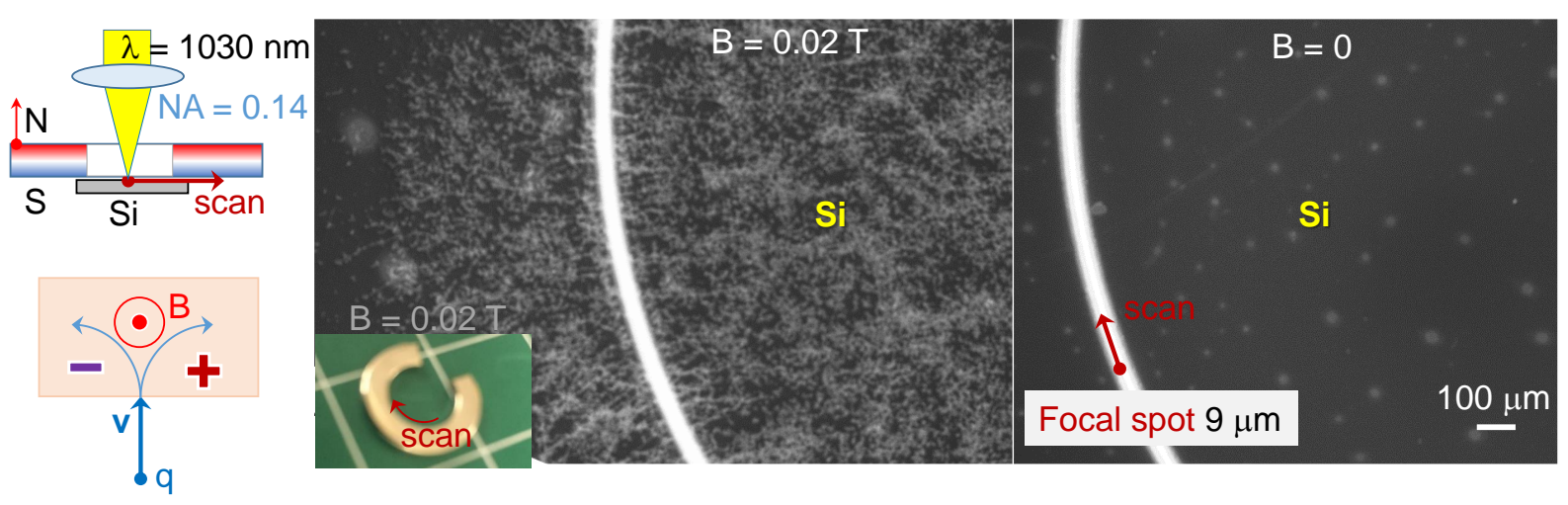

Figure 6. Debris formation for ablation of Si sample with a $B=0.02 \mathrm{~T}$ magnet placed on it. Laser was scanned along the internal rim of the magnet at constant distance. For the $B=0$ case, the same size and shape non-magnetic material was used. Conditions: scan speed $v_{s}=100 \mu \mathrm{m} / \mathrm{s}$, constant density mode with 250 pulses $/ \mu \mathrm{m}$, pulse energy $E_{p}=25 \mathrm{~nJ}(T=0.82$ transmission of the objective lens; $N A=0.14)$, laser repetition rate $f=200 \mathrm{kHz}$. Experimental geometry and charge separation due to Lorentz force are schematically shown.

\section{Discussion}

\subsection{Surface plasmon polariton}

The surface plasmon polariton (SPP) wave at the Si-air interface can be launched when the energy and momentum conservation laws are satisfied. The phase matching condition for the SPP wavevector $k^{s p p}=k_{1}^{s p p}+i k_{2}^{s p p}$ is given by:

$$
\begin{gathered}
k_{1}^{s p p}=k_{0} \sqrt{\frac{\varepsilon_{1}^{S i} \varepsilon_{1}^{a i r}}{\varepsilon_{1}^{S i}+\varepsilon_{1}^{a i r}}}, \\
k_{2}^{s p p}=k_{0}\left(\sqrt{\frac{\varepsilon_{1}^{S i} \varepsilon_{1}^{a i r}}{\varepsilon_{1}^{S i}+\varepsilon_{1}^{a i r}}}\right)^{3} \times \frac{\varepsilon_{2}^{S i}}{2\left(\varepsilon_{1}^{S i}\right)^{2}},
\end{gathered}
$$

where $\varepsilon^{S i} \equiv \varepsilon_{1}^{S i}+i \varepsilon_{2}^{S i}=12.709+i 1.7146 \times 10^{-3}$ is the permittivity of $\operatorname{Si}$ at $\lambda=1030$ nm wavelength [23], $k_{0}=2 \pi / \lambda$ is the wavevector in free space, $\varepsilon^{a i r} \equiv \varepsilon_{1}^{a i r}=1$ is the permittivity of air. The SPP wavelength $2 \pi / k_{1}^{s p p}=1.07 \mu \mathrm{m}$ (Eqn. 1$)$ and propagation length is $1 /\left(2 k_{2}^{s p p}\right)=17.3 \mathrm{~mm}$ (Eqn. 2).

When the electron density in $\mathrm{Si}$ increases during laser pulse irradiation, the real part of permittivity $\varepsilon_{1}^{* S i}\left(*\right.$ marks photo-excited state) decreases and imaginary part $\varepsilon_{2}^{* S i}$ increases due to free carrier absorption. The dielectric breakdown is defined by $\varepsilon_{1}=0$. A laser-induced air breakdown occurs at 
electrical field $E_{0}=3 \times 10^{6} \mathrm{~V} / \mathrm{m}$, and at the utilised irradiation conditions, it is reached at a point above the sample surface. This influences the amount and the area of the light energy deposition on the surface of Si. Only at lower pulse energies did the laser ablation pits have diameters similar to that of the focal spot $1.8 \mu \mathrm{m}$ (Figure 4(a)). Numerical modeling of linear intensity with geometrical locations of the focus on the Si surface (Figure 4(b)) shows a higher intensity of light field $E^{2}$ above the samples surface due to interference with the back-reflected light. There an initiation of air breakdown as expected.

An increase of the period between the ablated pits for smaller gap $x$ (a stronger $B$-field) can be explained by ionisation of air or/and Si. Indeed, when $\varepsilon_{1}^{* a i r}=0.75 \mathrm{in}$ Eqn. 1, the period $\Lambda_{s}=$ $2 \pi / k_{1}^{s p p}=1.22 \mu \mathrm{m}$ as observed for the larger gaps between magnets $x=4-5 \mathrm{~mm}$ (Figure 2(a)).

The line of ablation pits formed by the SPP had a period dependent on the B-field strength $(x$ gap) and its orientation always followed the magnetic field lines $N \rightarrow S$. Reduction of the $\varepsilon_{1}^{* S i}$ of photo-excited Si [24] can explain the increase of $\Lambda_{s}$ due to air breakdown which directly follows from Eqn. 1. Variation of $\varepsilon_{1}^{* S i}$ with $B$ should be caused by the different electron density $N_{e}$, which are proportional to the absorbed energy defined by the absorbance $A=1-R ; R$ is the reflectivity coefficient. The absorbed energy density $\left[\mathrm{J} / \mathrm{cm}^{3}\right]$ at the end of the laser pulse $W_{a}=2 A F_{p} / l_{a b s}$ [25], where the fluence $F_{p}=\int_{0}^{t_{p}} I_{p}(t) d t$, the absorption depth for the E-field is $l_{a b s}=c /(\omega k)=2 \pi / \kappa$ and is dependent on the refractive index $\sqrt{\varepsilon}=n+i \kappa$ and $A=4 n /\left[(n+1)^{2}+\kappa^{2}\right]$; the depth of absorption is $l_{a b s} / 2$ since $I \propto E^{2}[26]$.

The electron density $N_{e}$ at the end of the pulse is defined by $\int_{0}^{t_{p}} I_{p}(t) d t$ as described above. The average intensity of the laser pulse is $I_{a}=c \varepsilon_{0} E_{0}^{2} / 2 \equiv c B_{0}^{2} /\left(2 \mu_{0}\right) \equiv E_{0} B_{0} /\left(2 \mu_{0}\right)$ where $E_{0}$ is the amplitude of the electrical field strength $[\mathrm{V} / \mathrm{m}], B_{0}=E_{0} / c$ is the maximum magnetic field strength $[\mathrm{T}], \varepsilon_{0}, \mu_{0}$ are the permittivity and permeability of the free space, respectively. Generation of electrons by laser pulse follows in time the instantaneous intensity $I \propto E B$, where the light's field $B_{h v} \approx 60 \mathrm{~T}\left(E_{p}=1.13 \mu \mathrm{J} ;\right.$ Fig. 1$)$ while $\mathrm{Nd}_{2} \mathrm{Fe}_{14} \mathrm{~B}$ magnets added a $B_{\text {ext }}=0.1 \mathrm{~T}$, which was a negligible contribution; here $h v$ marker is for the light field. The dependence of $\Lambda_{s}$ period with $B$-field strength as well as the alignment $N \rightarrow S$ of the pattern should be linked to the plasma density at the end of the pulse (defining $\varepsilon_{1}^{* S i}$ ) and ensuing plasma dynamics in the external B-field (after the light pulse). Due to the vectorial nature of Lorentz force, electrons are coiled around the magnetic field lines with different cyclotron frequency and rotation radius.

Polarisation of laser pulses can define the orientation of oscillation in electrons - the velocity vector $\mathbf{v}_{e}$ - in the applied light field $\mathbf{v}_{e} \| \mathbf{E}$ was not an important factor for formation of SPP-like pattern.

The phase mismatch of the SPP wavevector $k_{1}^{s p p}=5.87 \times 10^{6} \mathrm{~m}^{-1}$ with that of the laser light $k_{0}=6.1 \times 10^{6} \mathrm{~m}^{-1}$ is accommodated by scattering and redirection from strongly localised scattering point, the $d=1.8 \mu \mathrm{m}$ diameter focal spot, from which light is scattered into wide range of angles $\theta_{s}$. The condition $k_{1}^{s p p}=k_{0} \pm \cos \theta_{s} \times(2 \pi / d)$ would launch SPP on the surface of photo-excited Si with the period defined by its permittivity and direction governed along the direction of the external B-field; here $2 \pi / d=3.5 \times 10^{6} \mathrm{~m}^{-1}$. The external field was contributing to the absorbed light energy in a pre-surface skin layer of $\mathrm{Si}$ of $1 / l_{a b s}$ depth as discussed above.

\subsection{B-field generation}

At high laser intensity, energy deposition into the skin (absorption) depth becomes sub-wavelength. This favors formation of strong electron concentration and temperature gradients and can lead to spontaneous generation of magnetic field [27]. When the electron temperature gradient $\nabla T_{e}$ and the concentration gradient $\nabla N_{e}$ are not parallel, $\partial \mathbf{B} / \partial t=\left[\nabla T_{e} \times \nabla N_{e}\right] /\left(e N_{e}\right)$ [27]. Plasma dynamics immediately after the laser pulse is influenced by the externally applied B-field as discussed for the debris formation, however, in Si it caused SPP-like aligned ablation micro-pits. The stronger B-field caused a larger period $\Lambda_{s}$ for the same laser pulse energy (Figure 5). We used low-conductivity $\mathrm{Si}$ and the photo-generated carrier dynamics starts from the optically excited volume with a lateral 
cross section $\sim 2 \mu \mathrm{m}$ and few micrometers deep. Further studies are planned for determination of the mechanism of SPP-like ablation patterns.

\section{Materials and Methods}

Direct laser writing was carried out with laser fabrication integrated system (Altechna, Ltd.) comprised of $t_{p}=230 \mathrm{fs}$ pulse duration and $\lambda=1030 \mathrm{~nm}$ wavelength pulsed laser (Pharos, Light Conversion, Ltd.) and high precision mechanical stages (Aerotech, $\mathrm{GmbH}$ ). All laser fabrication was carried out in air (Cleanroom, Class 1000). The laser beam profile was measured utilising a Ophir Spiricon SP928 Beam profiling camera.

The magnet and sample holding jigs with variable magnet spacing were 3D printed (Stratasys Objet260) in FullCure 720 using the PolyJet process in high resolution mode (16 $\mu$ m layer height). The depth of the sample area was designed to ensure the silicon surface remained at the same height as the circular magnet's central axis.

The standard phosphorus-doped (n-type) c-Si $\langle 100\rangle$ was utilised for ablation with an external magnetic $\mathbf{B}$ field usually applied using a 3D printed plastic jig (housing 5-mm-diameter $\mathrm{Nd}_{2} \mathrm{Fe}_{14} \mathrm{~B}$ magnets, up to four each side) with B-field applied parallel to Si-sample's surface. Also, different diameter and thickness neodymium magnets were used for application of B-field perpendicular to Si-sample's surface. The B-field strength was directly measured and was ranging from $B_{\text {ext }}=0.03$ to 0.16 Tesla. The conductivity of n-Si was measured by the van der Pauw method with Ecopia HMS-3000. It confirmed the n-type conductivity of $\sigma=3.76 \times 10^{-4} \Omega^{-1} / \mathrm{cm}$ (resistivity of $\rho \equiv 1 / \sigma=2.658 \times$ $10^{3} \Omega \mathrm{cm}$ ), bulk concentration of carriers $n_{e}=2.546 \times 10^{13} \mathrm{~cm}^{-3}$, mobility of $\mu_{e}=92.25 \mathrm{~cm}^{2} /$ V.s and sheet resistance of $5.31 \times 10^{4} \Omega / \square$. There was no difference observed for the tested low conductivity intrinsic and $\mathrm{n}-/ \mathrm{p}$-type $\mathrm{Si}$ in terms of formation of the ablation pattern.

All samples were cleaned in acetone/IPA prior to use to minimise surface contamination, prior to fabrication by direct laser writing. Ablation conditions varied from single pulses to heavly overlapping pulses, of low and high energy (1-1000 nJ pulses), with objective lenses of numerical aperture $N A=$ 0.14 and 0.7 (Mitutoyo). Pulse energy was measured at the entrance of an objective lens together with measured transmission coefficient $T$. Laser ablation was carried out at irradiance well exceeding the ablation threshold [28].

Strength of magnetic field was measured with a Gauss probe (Xplorer GLX PS-2002 with 2 axis Magnetic Field sensor, Pasco) with several aligned magnets like that seen in the utilised sample holders. A scanning electron microscopy (SEM) was used to characterise surfaces of laser ablated regions (Raith EBL $150^{\text {TWO }}$, Raith).

\section{Conclusions and outlook}

It is shown that by applying magnetic B-field of $B \sim 0.1 \mathrm{~T}$ across a 5-to-2 $\mathrm{mm}$ gap with a $\mathrm{Si}$ sample in it, the field of ablation debris as well as surface morphology of the ablation pit are strongly affected. The smaller the gap (stronger B-field), the larger the period between the micro-ablation pits were observed along the $N \rightarrow S$ direction of the externally applied magnetic fields. The period of this linear pattern was not dependent on the polarisation of the incident light but was guided by B-field orientation.

Future studies will be focused on determination of the exact mechanism of periodic pattern formation. Externally applied electric and magnetic fields can provide vectorial control of light matter interaction via the Lorentz force of photo-excited/generated carriers (electrons, holes, ions) and enrich the available control toolbox of light-matter interactions. Engineering of permittivity via optically induced/controlled dielectric-to-metal (Die-Met) [29] transition with ultra-short laser pulses is becoming new method to structure materials.

Author Contributions: Conceptualization, S.J.; methodology, J.M., S-H.N.; validation, J.M., T.K. S-H.N.; experiments, J.M., S-H.N., T.K. B.C.C.C.; data analysis, all the authors; writing-review and editing, all the authors. 
Funding: This research was funded by the Australian Research Council Discovery projects DP170100131 and DP190103284.

Acknowledgments: SJ is grateful to Workshop of Photonics (WOP), Lithuania, for a technology transfer project which was instrumental to establish the femtosecond laser fabrication facility at Swinburne. This work was performed at the Nanofabrication Facility - Nanolab at Swinburne. We are grateful to Prof. E.G. Gamaly for discussion and critical comments. This work was performed in part at the Melbourne Centre for Nanofabrication $(\mathrm{MCN})$ in the Victorian Node of the Australian National Fabrication Facility (ANFF).

Conflicts of Interest: The authors declare no conflict of interest.

\section{References}

1. Davis, K.M.; Miura, K.; Sugimoto, N.; Hirao, K. Writing waveguides in glass with a femtosecond laser. Opt. Lett. 1996, 21, $1729-1731$.

2. Ròdenas, A.; Gu, M.; Corrielli, G.; Paié, P.; John, S.; Kar, A.; Osellame, R. Three-dimensional femtosecond laser nanolithography of crystals. Nature Photonics 2019, 13, $105-109$.

3. Watanabe, M.; Juodkazis, S.; Matsuo, S.; Nishii, J.; Misawa, H. Cross-Talk in Photoluminescence Readout of Three-Dimensional Memory in vitreous Silica by One- and Two-Photon Excitation. Jpn. J. Appl. Phys. 2000, 39, 6763-6767.

4. Juodkazis, S.; Matsuo, S.; Misawa, H.; Mizeikis, V.; Marcinkevicius, A.; Sun, H.B.; Tokuda, Y.; Takahashi, M.; Yoko, T.; Nishii, J. Application of femtosecond laser pulses for microfabrication of transparent media. Appl. Surf. Sci. 2002, 197-198, 705-709.

5. Shimotsuma, Y.; Kazansky, P.; Qiu, J.; Hirao, K. Self-organized nanogratings in glass irradiated by ultrashort light pulses. Phys. Rev. Lett. 2003, 91, 247405/1-4.

6. Umran, F.A.; Liao, Y.; Elias, M.M.; Sugioka, K.; Stoian, R.; Cheng, G.; Cheng, Y. Formation of nanogratings in a transparent material with tunable ionization property by femtosecond laser irradiation. Opt. Express, $21,15259-15267$.

7. Ozkan, A.; Malshe, A.; Railkar, T.; Brown, W.; Shirk, M.; Molian, P. Femtosecond laser-induced periodic structure writing on diamond crystals and microclusters. Applied Physics Letters 1999, 75, 3716.

8. Das, S.K.; Messaoudi, H.; Debroy, A.; McGlynn, E.; Grunwald, R. Multiphoton excitation of surface plasmon-polaritons and scaling of nanoripple formation in large bandgap materials. Opt. Mat. Express 2013, 3, $1705-1715$.

9. Juodkazis, S.; Nishimura, K.; Misawa, H.; Ebisui, T.; Waki, R.; Matsuo, S.; Okada, T. Control over the State of Crystallinity: Sapphire. Adv. Mat. 2006, 18, $1361-1364$.

10. Gamaly, E.E.; Juodkazis, S.; Nishimura, K.; Misawa, H.; Luther-Davies, B.; Hallo, L.; Nicolai, P.; Tikhonchuk, $\mathrm{V}$. Laser-matter interaction in a bulk of a transparent solid: confined micro-explosion and void formation. Phys. Rev. B 2006, 73, 214101.

11. Yamaguchi, M.; Ueno, S.; Kumai, R.; Kinoshita, K.; Murai, T.; Tomita, T.; Matsuo, S.; Hashimoto, S. Raman spectroscopic study of femtosecond laser-induced phase transformation associated with ripple formation on single-crystal SiC. Applied Physics A: Materials Science 2010, pp. 1-5.

12. Drs, J.; Kishi, T.; Bellouard, Y. Laser-assisted morphing of complex three dimensional objects. Optics Express 2015, 23, 17355-17366.

13. Marcinkevicius, A.; Juodkazis, S.; Watanabe, M.; Miwa, M.; Matsuo, S.; Misawa, H.; Nishii, J. Femtosecond Laser-Assisted Three-Dimensional Microfabrication in Silica. Opt. Lett. 2001, 26, 277-279.

14. Yamasaki, K.; Juodkazis, S.; Matsuo, S.; Misawa, H. Three-dimensional microchannels in polymers: one step fabrication. Appl. Phys. A 2003, 77, 371-373.

15. Juodkazis, S.; Yamasaki, K.; Mizeikis, V.; Matsuo, S.; Misawa, H. Formation of Embedded Patterns in Glasses Using Femtosecond Irradiation. Appl. Phys. A 2004, 79, 1549 - 1553.

16. Zhang, D.; B Gokc and, S.B. Laser synthesis and processing of colloids: fundamentals and applications. Chemical Reviews 2017, 117, 3990 - 4103.

17. Oktem, B.; Pavlov, I.; Ilday, S.; Kalaycioglu, H.; Rybak, A.; Yavas, S.; Erdogan, M.; Ilday, F.O. Nonlinear laser lithography for indefinitely large-area nanostructuring with femtosecond pulses. Nat. Photonics 2013, 7,897-901. 
18. Bhuyan, M.K.; Courvoisier, F.; Lacourt, P.A.; Jacquot, M.; Salut, R.; Furfaro, L.; Dudley, J.M. High aspect ratio nanochannel machining using single shot femtosecond Bessel beams. Appl. Phys. Lett. 2010, 97, 081102.

19. Wang, G.; Yu, Y.; Jiang, L.; Li, X.; Xie, Q.; Lu, Y. Cylindrical shockwave-induced compression mechanism in femtosecond laser Bessel pulse micro-drilling of PMMA. Appl. Phys. Lett. 2017, 110, 161907.

20. Osellame, R.; Hoekstra, H.; Cerullo, G.; Pollnau, M. Femtosecond laser microstructuring: an enabling tool for optofluidic lab-on-chips. Laser Photonics Rev. 2011, 5, 442-463.

21. Iwase, H.; Kokubo, S.; Juodkazis, S.; Misawa, H. Suppression of ripples on Ni surface via a polarization grating. Opt. Express 2009, 17, 4388-4396.

22. Buividas, R.; Gervinskas, G.; Tadich, A.; Cowie, B.C.C.; Mizeikis, V.; Vailionis, A.; de Ligny, D.; Gamaly, E.G.; Rode, A.V.; Juodkazis, S. Phase Transformation in Laser-Induced Micro-Explosion in Olivine $(\mathrm{Fe}, \mathrm{Mg})_{2} \mathrm{SiO}_{4}$. Adv. Eng. Mat. 2014, 16, 767 - 773.

23. Schinke, C.; Peest, P.C.; Schmidt, J.; Brendel, R.; Bothe, K.; Vogt, M.R.; Kröger, I.; Winter, S.; Schirmacher, A.; Lim, S.; Nguyen, H.T.; MacDonald, D. Uncertainty analysis for the coefficient of band-to-band absorption of crystalline silicon. AIP Advances 2015, 5, 67168.

24. Sokolowski-Tinten, K.; von der Linden, D. Generation of dense electron-hole plasmas in silicon. Phys. Rev. B 2000, 61, $2643-2650$.

25. Gamaly, E.G.; Rode, A.V. Physics of ultra-short laser interaction with matter: From phonon excitation to ultimate transformations. J. Progr. Quant. Electron. 2013, 37, 215-323.

26. Gamaly, E.G.; Rode, A.V.; Luther-Davies, B. Ablation of solids by femtosecond lasers: Ablation mechanism and ablation thresholds for metals and dielectrics. Physics of Plasmas 2002, 18, 949 - 957.

27. Haines, M.G. Saturation Mechanisms for the Generated Magnetic Field in Nonuniform Laser-Matter Irradiation. Phys. Rev. Lett. 1997, 78, 254- 257.

28. Wang, C.; Huo, H.; Johnson, M.; Shen, M.; Mazur, E. The thresholds of surface nano-/micro-morphology modifications with femtosecond laser pulse irradiations. Nanotechnology 2010, 21, 75304.

29. Gamaly, E.G.; Rode, A.V. Ultrafast re-structuring of the electronic landscape of transparent dielectrics: new material states (Die-Met). Appl. Phys. A 2018, 124, 278(1-11). 


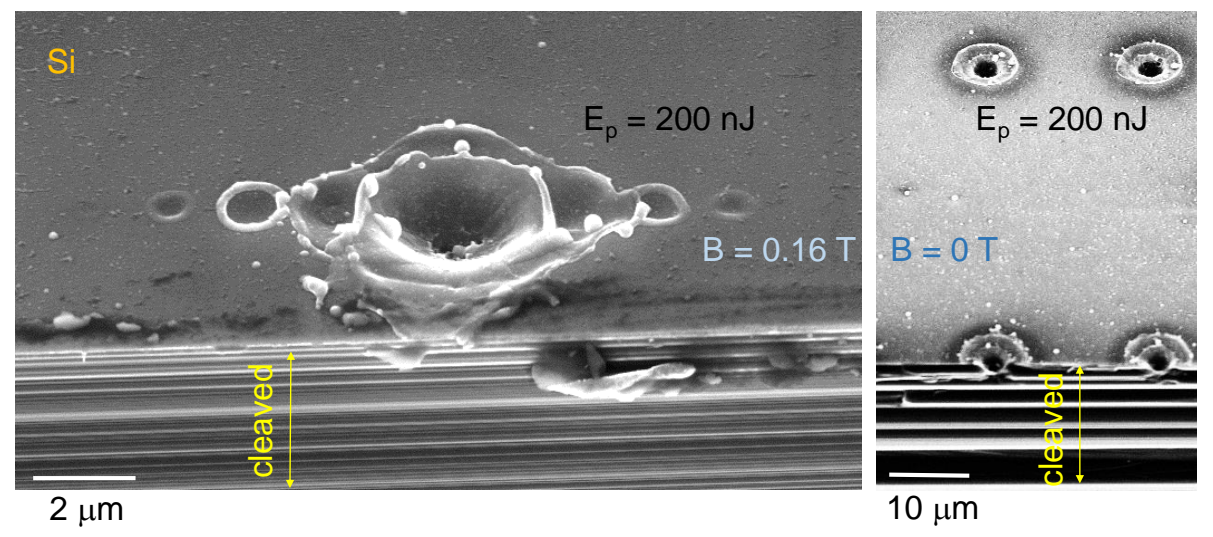

Figure A1. $45^{\circ}$ Slanted-view SEM images of the ablation sites made by $E_{p}=200 \mathrm{~nJ}$ energy single pulse (objective lens $N A=0.7, T=0.22$ ) with magnetic field and without. The linear pattern of micro-ablation pits is aligned with the B-field direction.
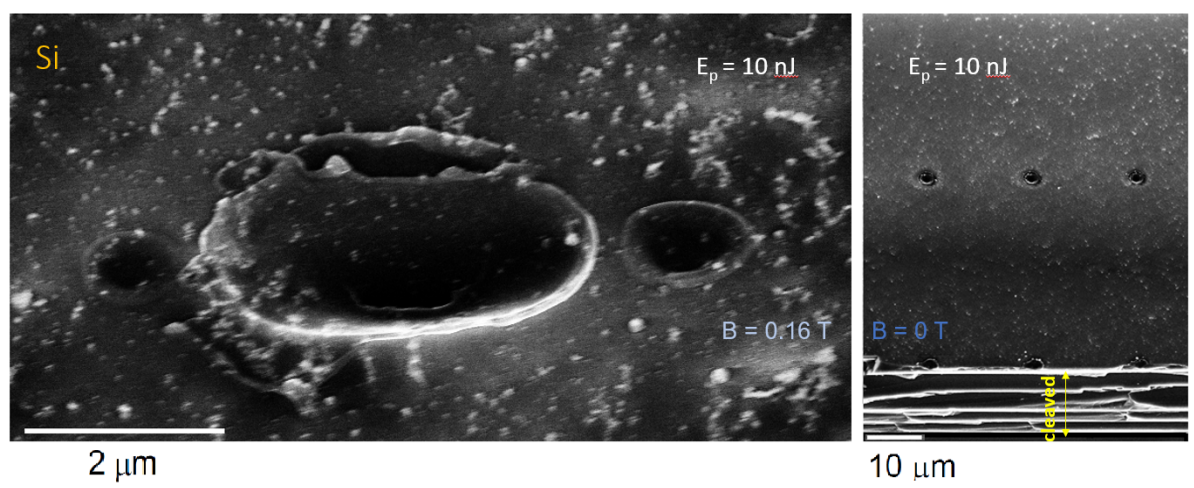

$10 \mu \mathrm{m}$

Figure A2. $45^{\circ}$ Slanted-view SEM images of the ablation sites made by $E_{p}=10 \mathrm{~nJ}$ energy single pulse (objective lens $N A=0.7, T=0.22$ ) with magnetic field and without. The linear pattern of micro-ablation pits is aligned in the B-field direction.

\section{Appendix A Supplement}

Tilted view SEM images of the typical ablated single-pulse ablated pulses are shown in Figure A1. 